\title{
High EEG-gamma-power codes perceptual states of ambiguous motion
}

\author{
Joscha T Schmiedt*1,2, David Rotermund ${ }^{1}$, Canan Basar-Eroglu ${ }^{2}$ and \\ Klaus Pawelzik ${ }^{1}$
}

\begin{abstract}
Address: ${ }^{1}$ Institute for Theoretical Physics, Universität Bremen, Bremen, 28344, Germany and 2Institute of Psychology and Cognition Research, Universität Bremen, Bremen, 28344, Germany

Email: Joscha T Schmiedt* - schmiedt@uni-bremen.de

* Corresponding author
\end{abstract}

from Eighteenth Annual Computational Neuroscience Meeting: CNS*2009

Berlin, Germany. 18-23 July 2009

Published: 13 July 2009

BMC Neuroscience 2009, 10(Suppl I):P72 doi:10.1 I86/I47I-2202-10-SI-P72

This abstract is available from: http://www.biomedcentral.com/I47I-2202/I0/SI/P72

(c) 2009 Schmiedt et al; licensee BioMed Central Ltd.

It has been shown that the perceptual experience of a viewer can be tracked using multivariate analysis on noninvasive functional magnetic resonance imaging (fMRI) data. The resulting time series of three-dimensional images related to brain activity have successfully been classified using machine learning methods like Support Vector Machines (SVM) [1]. In addition, it is possible to distinguish cognitive states, such as the two possible perspectives in binocular rivalry as in [2].

Based on these findings, this study aims at investigating whether it is possible to decode the bistable perception of a human viewer on a single trial basis using thirty channels of electroencephalographic (EEG) data. For this purpose, we classify the direction of motion of the stroboscopic ambiguous motion (SAM) pattern, which is known to be functionally related to gamma-band power [3]. Taking advantage of the temporal resolution of EEG data, we use SVMs that operate in the time-frequency domain in order to study the oscillatory coding of an ambiguous visual stimulus in the brain.

Our results show that it is possible to detect the direction of motion with accuracy significantly above chance level. The best classification performance is reached using high frequency gamma-band power, which suggests a perceptrelated synchronization similar to [4]. This demonstrates that dynamical mechanisms underlying specific mental contents in the human brain can be studied using modern machine learning methods in contrast to conventional EEG research which focuses on spatially and temporally localizing cognitive features.

\section{Acknowledgements}

Thanks to Birgit Mathes and Juliane Bagdasaryan for the help in conducting the experiments.

\section{References}

I. Kamitani $Y$, Tong F: Decoding the visual and subjective contents of the human brain. Nat Neurosci 2005, 8:679-685.

2. Haynes JD, Rees G: Predicting the stream of consciousness from activity in human visual cortex. Curr Biol 2005, I5:I30I-I307.

3. Basar-Eroglu C, Strüber D, Kruse P, Basar E, Stadler M: Frontal gamma-band enhancement during multistable visual perception. Int J Psychophysiol 1996, 24: I I3-1 25.

4. Kreiter AK, Singer W: Stimulus-dependent synchronization of neuronal responses in the visual cortex of the awake macaque monkey. J Neurosci 1996, 16:2381-2396. 